\title{
CAPÍTULO XI
}

\section{Condiciones para la difusión de los sistemas fotovoltaicos residenciales en Colombia}

Carlos Fernando Morales Sánchez*

* Docente investigador Programa de Finanzas y Comercio Internacional, Universidad de La Salle, Bogotá DC, Colombia. Economista y Magíster en Ciencias Económicas de la Universidad Nacional de Colombia. 


\section{Introducción}

Según el Grupo intergubernamental de expertos sobre el cambio climático (IPCC, por sus siglas en inglés), las fuentes de energía convencionales tales como el petróleo y el carbón, generan emisiones en el planeta causantes del efecto invernadero, así como emisiones de otros gases que producen riesgos para la salud humana (IPCC, 2014). Adicionalmente, la escasez de estos recursos, la elevada incertidumbre en los procesos de prospección y su elevada concentración geográfica, se han traducido en elevados costos, baja confiabilidad en la oferta y tensiones geopolíticas.

Como respuesta a esta problemática, ha venido creciendo el uso de energías alternativas tales como la energía solar fotovoltaica, la energía eólica y la biomasa, entre otras. La Agencia Internacional de Energía (IEA) referencia varios beneficios sociales tales como: reducción en el precio de la energía eléctrica derivado de un aumento en la oferta y confiabilidad del servicio (fenómeno conocido como merit order effect), contribución a la seguridad energética de los países, generación de empleos locales para la instalación y mantenimiento de sistemas descentralizados, fortalecimiento de la seguridad energética y reducción de la contaminación (IEA, 2014). A pesar de la existencia de estos beneficios, el proceso de tránsito hacia las fuentes alternativas es complejo porque los costos de generación usualmente han sido más elevados que los de las fuentes convencionales. De hecho, aun cuando esta diferencia en costos se ha venido reduciendo, persiste una dificultad de fondo: el reto de cambiar todo el paradigma tecnológico asociado los combustibles fósiles como fuente de energía motriz para automóviles, barcos y aviones y fuente de energía eléctrica para diversos usos (Pérez, 2004).

Una posible respuesta al problema del paradigma tecnológico se ha planteado desde el esquema de generación distribuida (GD) el cual consiste en la provisión de energía eléctrica a partir de la producción por parte de pequeñas unidades descentralizadas cercanas a los lugares de consumo. Bajo el esquema de GD ha florecido en varios países del mundo, un mercado de pequeños sistemas de generación usando energías renovables, en el que empresas, comercios y viviendas pasan de ser únicamente consumidores a también producir 
energía, borrando los límites entre consumo y producción; de ahí, que se considere éste como un ejemplo del prosumidor anticipado por McLuhan (Rusko, 2012). La energía generada de esta forma puede, o bien ser consumida directamente por el pequeño generador, o entregarse al sistema interconectado tradicional si se cuentan con medidores inteligentes que registren la generación de excedentes. El esquema de generación distribuida usando fuentes de energía limpia tiene también otra ventaja: aunque los costos fijos de la inversión son relativamente elevados, el costo marginal de la generación y distribución de una adicional de energía es prácticamente cero (Rifkin, 2014).

Varios gobiernos alrededor del mundo han diseñado incentivos para las energías renovables bajo esquemas de generación distribuida. El caso que aquí se estudia es el de los sistemas de generación solar fotovoltaica usando un incentivo conocido como tarifas de alimentación o feed-in tariffs (FIT). Este incentivo ha sido bastante exitoso en términos de su difusión; es así, como se ha implementado en más de 30 países como Alemania, Estados Unidos y China (Sun y Nie, 2015). La idea detrás de estas tarifas es que cuando cualquier unidad económica (incluso familias) genere energía eléctrica proveniente de fuentes renovables, se le pague un precio preferencial que incentive una mayor generación y compense adecuadamente los efectos sociales positivos (externalidades) que estas fuentes ofrecen.

En el caso de Colombia, a pesar que tiene un elevado potencial de generación de energía renovable empleando fuentes como el sol y el viento (Paredes y Ramírez, 2017), no se han implementado incentivos similares a las tarifas de alimentación, ni parecen tener un lugar relevante en la discusión pública. Aunque se han realizado cambios en la normatividad, aún persisten vacíos que no permiten a las empresas del sector de las energías renovables, realizar inversiones con seguridad jurídica e igualdad de condiciones frente a los productores tradicionales. Por ejemplo, la ley 1715 de 2014, establece un marco legal para la promoción de energías no convencionales renovables y acepta la posibilidad de esquemas de generación distribuida definiendo a los prosumidores de energía como auto generadores. Sin embargo, no se ha definido una reglamentación sobre la forma en que se pagan excedentes ni mucho menos sobre eventuales tarifas de alimentación para la generación de energía bajo esquemas de generación distribuida (Saenz, 2017). 
Una de las razones para el escaso impulso de las energías renovables en Colombia radica en su estructura de generación eléctrica: Colombia aporta solo el 0,42\% de las emisiones mundiales de dióxido carbono (IDEAM, PNUD, MADS, DNP, CANCILLERÍA, 2016). Además, de acuerdo a la información de la Compañía Expertos en Mercados (XM) sobre la gestión del sistema eléctrico, cerca del 69,93\% de la energía el país proviene de las hidroeléctricas, las cuales generan emisiones considerablemente menores (XM, 2017). Sin embargo, el uso de hidroeléctricas no está exento de problemas. Esta tecnología se caracteriza por elevados costos fijos, poca flexibilidad ante cambios como los que produce el cambio climático y grandes impactos socio-ambientales localizados en las regiones donde se efectúan estos proyectos (McCartney, 2009). Sin embargo, dado que este potencial se desaprovecha, es necesario estudiar las razones por las cuales en Colombia no se difunde el uso de sistemas de energía solar fotovoltaica analizando particularmente, el caso de los auto generadores bajo un esquema de tarifas de alimentación.

\section{La problemática de la energía en San Andrés y Providencia}

Un caso relevante es el del departamento de San Andrés y Providencia que por su carácter insular tiene una estructura de generación de energía diferente al resto de Colombia. Aparte de algunas excepciones sobre las cuales no hay registro, la energía eléctrica se genera mediante generadores diésel ubicados en la planta Evans (Unidad de Planeación Minero Energética UPME, 2015). Este modo de generación es altamente contaminante pues implica la emisión de 134 mil toneladas de dióxido de carbono al año (Gómez, Afanador, Rodríguez, 2016). Además, la combustión del diésel genera otras emisiones altamente tóxicas como es el caso del dióxido de azufre. Al mismo tiempo, esta fuente resulta bastante costosa: según el Sistema Único de Información de Servicios Públicos Domiciliarios (SUI) el costo del kilovatio hora en San Andrés en el 2017 es de 830 pesos, el cuál es casi el doble del valor en ciudades continentales como Cartagena o Bogotá (SUI, 2017).

Si se tiene en cuenta que la tarifa media percibida por los usuarios es de 300 pesos el kilovatio hora, el resultado es que el Estado colombiano está financiando más del 60\% del valor de la energía consumida en el departamento. Según el Banco Interamericano de De- 
sarrollo (BID), este subsidio es de alrededor de 25 millones de dólares anuales, lo cual representa alrededor del $40 \%$ de los subsidios que el Estado colombiano proporciona para ofrecer energía a las Zonas No Interconectadas (ZNI) del país (Gómez et al., 2016). Además de la carga fiscal, existe el riesgo de que las condiciones bajo las cuales se ofrece el subsidio, generen efectos negativos respecto al uso de la energía en el departamento. Por ejemplo, un informe de auditoría realizado por la Contraloría General de la República (2012) sobre la concesión del servicio de energía en el departamento, advierte que la decisión del gobierno colombiano de aumentar el máximo consumo energético subsidiado de 400 a 800 kWh por mes (cuando el consumo promedio residencial en Colombia es de $200 \mathrm{kWh} / \mathrm{mes}$ ) no estimula el desarrollo de una cultura del ahorro y la eficiencia del servicio.

Este mismo informe de auditoría señala serios problemas de planeación y de uso de recursos públicos destinados a fortalecer la generación de energía eléctrica en el departamento. La Sociedad Productora de Energía de San Andrés y Providencia (SOPESA S.A. E.S.P.), empresa a cargo de la generación, está comprometida a construir y poner en marcha una planta generadora a partir de la incineración de residuos sólidos urbanos (RSU) y un parque de generación de energía eólica. La planta RSU se construyó con capacidad para proveer hasta un megavatio de potencia ( $1 \mathrm{MW})$, pero aún no ha entrado en funcionamiento por dos razones. Primero, debe implementarse antes un esquema de separación de basuras porque la planta RSU no puede incinerar todo tipo de residuos. Segundo, SOPESA no tiene el permiso para disponer de los residuos que se usarían en la planta (Contraloría General de la República, 2012). En cuanto al parque de generación eólica, no ha empezado a construirse porque la comunidad raizal no ha dado su permiso (Dinero, 2017).

Los problemas con estos proyectos de generación alternativa ponen en evidencia la poca legitimidad que tienen las actuaciones del Estado frente al tema energético. Esta falta de confianza también se explica por la no inclusión de gran parte de la población en las dinámicas económicas y políticas de la isla. El resultado, es que los raizales primero, perciben difícil el cambio hacia un sistema energético más eficiente y limpio; y segundo, ante eventuales ganancias de ese nuevo sistema, las comunidades tradicionales de la isla, suponen 
que difícilmente se verán beneficiadas (Guevara-Stone, Hawley, \& Tubb, 2016).

A pesar de estas dificultades, el departamento de San Andrés y Providencia tiene un elevado potencial para aprovechar la energía solar y del viento (Paredes y Ramírez, 2017). Su estatus como puerto libre, permite la importación de los equipos necesarios para los sistemas fotovoltaicos, sin el pago del impuesto al valor agregado (IVA) del 19\% que se paga en el resto de Colombia. Adicionalmente, hay casos en los que se ejecutan proyectos innovadores con éxito que pueden servir como programas piloto que sirven como experiencia para Colombia y otros países de la región. SOPESA contrató un programa para la implementación de un esquema de medición inteligente (net metering). Éste funciona a partir de medidores o contadores inteligentes que ofrecen información en tiempo real sobre generación y consumo, posibilitando identificar pérdidas en el sistema. Gracias al uso de medición inteligente, según reportes de SOPESA realizados a medios de comunicación (Dinero, 2017), se ha posibilitado reducir las pérdidas de energía del 31\% al 8\% de la generación. Otros beneficios son el mayor conocimiento y uso de la energía por parte del usuario y menores costos de comercialización (Gómez et al., 2016). Más aún, esta tecnología posibilita la provisión de energía bajo esquemas de generación distribuida con paneles fotovoltaicos.

\section{Fundamentos teóricos de la difusión de sistemas fotovoltaicos}

La tecnología de sistemas fotovoltaicos ya existe, pero el problema radica en la difusión tecnológica en un contexto como el de Colombia. Para estudiar los factores determinantes que pueden facilitar 0 dificultar la difusión de esta tecnología, se pueden emplear diversas corrientes de la teoría económica. En este trabajo, se discuten las perspectivas sobre la innovación que ofrecen Schumpeter (1939) y Rogers (2003), realizando una aproximación desde la economía institucional. Una perspectiva influyente, es la de la economía neoclásica y su supuesto de racionalidad económica. Desde este punto de vista, se podría considerar que si una tecnología nueva es superior a la convencional en términos de prestaciones o precio, los agentes económicos la adoptarían inmediatamente ya que sería la elección racional (Morrisson \& Potts, 2008). Es decir, no habría un proceso dinámico de adopción. Sin embargo, sin un proceso endógeno que 
explique el cambio tecnológico resulta difícil entender el desarrollo económico en una economía de mercado.

El concepto de destrucción creativa de Schumpeter representa una forma de reconocer el papel de la innovación en el desarrollo económico, pero al mismo tiempo, ayuda a entender los conflictos que genera. Aunque las innovaciones son determinantes para el desarrollo económico, su difusión destruye los sistemas anteriores que generaban valor y, por lo tanto, los grupos que se benefician de los sistemas precedentes buscarán dificultar el éxito de las nuevas tecnologías. Además, si las instituciones de una nación privilegian a estos grupos, será más difícil que se den las condiciones necesarias para que la innovación prospere. Este es justamente el caso de los países en vías de desarrollo, pues los beneficiarios de las instituciones tradicionales tienen condiciones más favorables para limitar los procesos de innovación resultando en un sistema económico poco adaptable y poco competitivo (Acemoglu, Johnson \& Robinson, 2005).

Reconocer la existencia de un proceso de difusión pasa por considerar tres dimensiones de análisis que afectan la decisión para individuos e instituciones: (i) Los costos de transacción en la adaptación, (ii) las limitaciones cognitivas que afectan la decisión de adopción y (iii) la influencia de las relaciones sociales en la decisión. Para estudiar estas dimensiones se emplea el marco de análisis de Everett Rogers (2003) sobre difusión de las innovaciones: su enfoque se sustenta en la psicología y la sociología para proponer cinco factores que afectan la posibilidad que un individuo o grupo adopte una nueva tecnología (Berkun, 2010). Estos según Ochoa y Peña (2012) son:

1. Ventaja relativa.

2. Compatibilidad.

3. Complejidad.

4. Posibilidad de experimentación.

5. Visibilidad.

Usando la clasificación de Rogers en el caso colombiano y en particular el del departamento de San Andrés y Providencia se encuentra se plantea el siguiente análisis: 
1. Ventaja relativa. Este factor se entiende como el grado en que una innovación ofrece mayor valor a sus predecesoras. En particular, es necesario que el uso de las instalaciones fotovoltaicas ofrezca una prerrogativa clara para los posibles adoptantes frente a las alternativas. Estas ventajas se pueden asociar a menores costos, mayor disponibilidad de energía o la posibilidad de rentabilizar los beneficios. Un ejemplo, es el del tamaño eficiente de la instalación: la pequeña escala de las instalaciones residenciales aumenta los costos fijos de la inversión inicial y, por lo tanto, el costo de la energía por kilovatio termina siendo muy elevado. Por consiguiente, la ventaja de usar sistemas fotovoltaicos depende de la escala. En el caso de San Andrés y Providencia, a diferencia de lo que sucede en la mayor parte del país, toda la carga de energía en el departamento, incluyendo estratos altos, sectores industrial y comercial, tiene un subsidio en la tarifa (Gómez et al., 2016). En consecuencia, desde el punto de vista de cualquier usuario de la región, la energía que se ofrece bajo el esquema actual ya es relativamente económica y es difícil que cualquier alternativa ofrezca un precio menor. Aquí aparece un dilema social. Mientras que la ventaja relativa puede no ser evidente a nivel individual, la ventaja si es considerable desde el punto de vista general. En otras palabras, hay externalidades positivas en la adopción de las energías renovables, que no son fácilmente apreciables por los agentes económicos individuales. En concreto, el hecho que toda la energía ofrecida sea subsidiada, y que la tarifa promedio sea baja, significa que desde el punto de vista individual, no se percibe un beneficio por cambiar a una tecnología que no representa considerables ahorros. Un dilema social similar tiene que ver con la gestión del riesgo. Para cada individuo es posible que no sea óptimo tomar riesgos con una tecnología nueva. Pero a nivel colectivo si aumentara el número de personas que la adoptaran, aunque algunos usuarios tuvieran pérdidas, el sistema de generación eléctrica se beneficiaría de una mayor confiabilidad (menor riesgo en la provisión) derivada de la diversificación de las fuentes y de la mayor disponibilidad de potencia. Adicionalmente, se reducirían los impactos ambientales negativos y el impacto de los subsidios en el presupuesto. En este caso, sería recomendable que se ofrecieran coberturas financieras a los adoptantes de forma que motiven la adopción de la tecnología. Esta propuesta es acorde a lo planteado por Kahneman (2011) en lo referente a las políticas frente al riesgo: se requiere un "marco ancho" que premie y proteja a quienes toman riesgos individuales para aumentar los efectos sociales positivos en el largo plazo. 
2. Compatibilidad. Se puede interpretar como el nivel de consistencia de la innovación con los valores, creencias, hábitos y estilos de vida de los posibles adoptantes. El proceso de hacer compatible la innovación con los paradigmas dominantes, se puede entender como un proceso de reducción de costos de transacción derivados de la adaptación (Dahlman, 1979). En particular, la generación de energías renovables bajo esquemas de generación distribuida implica profundos cambios regulatorios, considerables inversiones y la coordinación de varios actores lo cual implica fricciones con el esquema tradicional. Desde la perspectiva del Estado hay un problema de compatibilidad con el sistema regulatorio, pues los cambios sociales y tecnológicos se presentan con mayor velocidad que los cambios institucionales. Por ejemplo, las concesiones para la generación se realizan con plazos elevados, pero en el transcurso de la concesión, pueden aparecer tecnologías con mejor desempeño y por lo tanto las empresas que ganaron el derecho original perciben las nuevas tecnologías como competencia.

Otro factor a considerar es la infraestructura prexistente del sistema eléctrico. La incorporación sistemas fotovoltaicos mediante el esquema de generación distribuida requiere invertir en una estructura de medición inteligente (AMI) que permita mediante medidores inteligentes identificar si un auto generador consume o comparte energía. Dado que en San Andrés ya se viene implementando con éxito una estructura AMI, las posibilidades de adoptar sistemas fotovoltaicos son mayores que para el resto del país (Gómez et al., 2016). Otra dificultad radica en la infraestructura privada que deben tener los eventuales auto generadores; en particular, se requiere de condiciones adecuadas de infraestructura siendo las más representativas el espacio, la resistencia de la superficie (usualmente el techo) que soporte el peso del sistema y la facilidad del trabajo durante la instalación y el mantenimiento. Además, en la medida en que aumente el tamaño del sistema, la exigencia de los requerimientos de infraestructura tenderá a aumentar.

Por otra parte, los posibles adoptantes encuentran dificultades en el costo total de la inversión y la estructura financiera necesaria para llevarla a cabo. Según el SUI (2017), el 75\% de los usuarios, son de estratos 1 y 2. Por lo tanto, la disponibilidad de recursos para una inversión de que puede alcanzar los 7 mil dólares no es la óptima. Es por esta razón que la inversión en un sistema fotovoltaico se realiza 
a plazos y se buscan alternativas como el leasing (Liu, O'Rear, Tyner, \& Pekny, 2014). Finalmente se puede analizar la compatibilidad con elementos culturales. En el caso de varias regiones del país, la implementación de alternativas como la generación distribuida y la autogeneración implica mayor formalización de la provisión de energía, lo cual es incompatible con la costumbre de algunos habitantes que forman conexiones ilegales. Por el contrario, para algunas comunidades puede resultar atractivo el nivel de autonomía que se puede lograr con este tipo de instalaciones. Otro factor a considerar es la importancia que los posibles adoptantes le den a las problemáticas ambientales: si no se percibe que el calentamiento global o la contaminación son temas relevantes, se dificulta la difusión.

3. Complejidad. Es el grado de dificultad del aprendizaje necesario para poder usar la innovación. Hay varias dificultades en este sentido: el aprendizaje también representa un costo de transacción. Aclarar el concepto de generación distribuida y la forma en que un consumidor se puede convertir en auto generador ya implica un proceso complejo. Luego, es necesario explicar el funcionamiento de una instalación fotovoltaica bajo un esquema de generación distribuida. Finalmente, es preciso especificar los posibles beneficios y compararlos frente a la alternativa previamente existente y en este caso hay que incorporar consideraciones financieras. El componente financiero aumenta la complejidad de aprendizaje porque los beneficios de la inversión inicial no se ven inmediatamente y para ponerlos en evidencia se requieren de cálculos tales como el del valor presente neto, los cuáles no son fáciles de ejecutar o deducir. Desde el punto de vista del gobierno, un aprendizaje que resulta difícil de adquirir es el de la adecuada medición del riesgo respecto a la generación con el sistema tradicional. En concreto, el problema se puede ejemplificar con las implicaciones para el sistema de generación colombiano del fenómeno del niño en el periodo 2015 - 2016: la sequía que causó bajo nivel de los embalses y la falla de unidades de generación fundamentales mostró que el sistema tradicional no se encuentra lo suficientemente cubierto ante eventos climáticos extremos, precisamente el tipo de elementos que pueden ser más frecuentes por cuenta del cambio climático (Paredes y Ramírez, 2017). Una lección de este acontecimiento es que la provisión de energía para un país es un proceso complejo de elevada incertidumbre y por lo tanto, la planeación centralizada debe venir acompañada de una estructura distribuida que permita mayor adaptabilidad. 
4. Posibilidad de experimentación. Se refiere a la facilidad con la que se puede probar la innovación. Con frecuencia se necesitan simulaciones (con elevados componentes financieros) para demostrar la tecnología. Los prototipos, simulaciones y programas piloto reducen la incertidumbre sobre las implicaciones de la eventual adopción de una tecnología. Es decir, en la medida en que se pueda reducir la incertidumbre, se reducen los costos de transacción asociados a la decisión de usar una determinada tecnología (Kerr, Nanda \& Rhodes-Kropf, 2014). Adicionalmente, la experiencia asociada al aprendizaje mediante el uso (learning by using) es un factor que posibilita la reducción de costos asociados a los sistemas fotovoltaicos (Sandén, 2005). Sin embargo, en la práctica, no es fácil suponer que este tipo de tecnologías se puedan llevar a una casa temporalmente para probar si su funcionamiento responde a las expectativas. Por esta razón, se han realizado esfuerzos en el campo de las simulaciones tanto del funcionamiento como de las implicaciones financieras de los sistemas fotovoltaicos. Un importante antecedente es la iniciativa Project Sunroof de Google que ofrece información para Estados Unidos sobre instalación, funcionamiento y ahorros implicados por el uso de sistemas fotovoltaicos a nivel residencial. Ya hay una gran oferta de calculadoras en línea para instalaciones fotovoltaicas. Para el año 2017, el portal photovoltaic-software registra 18 programas de simulación en línea que ayudan en la toma de decisiones al respecto (Photovoltaic-software.com, 2017).

5. Visibilidad. Consiste en la facilidad con la que se puede mostrar o comunicar las ventajas de una innovación. Trabajos como el de Graziano y Gillingham (2015) han demostrado que el componente social es fundamental para facilitar la adopción de paneles fotovoltaicos a nivel residencial. En concreto, cuando hay varios vecinos usando esta tecnología aumenta la posibilidad de adoptarla. Es decir, el uso de la tecnología bajo condiciones geográficas (o espaciales) adecuadas que la hagan más visible y que facilitan la interacción social, tiene un efecto positivo en la difusión de las instalaciones fotovoltaicas. En consecuencia, si tomar una decisión sobre la base de criterios financieros es difícil, es posible que la influencia social gane peso como determinante para la difusión de esta tecnología. Por esta razón, la promoción de estas tecnologías depende una estrategia de comunicación que le de gran prioridad al voz a voz (Bass, 1969) dándole protagonismo a los mismos usuarios en el impulso y difusión de las instalaciones fotovoltaicas. 


\section{Metodología para el cálculo de tarifas de alimentación}

Para caracterizar la evolución de los costos de una instalación fotovoltaica y el valor de una tarifa de alimentación, se realiza una revisión documental usando como fuentes informes técnicos de instituciones tales como la Unidad de Planeación Minero-energética (UPME), el Banco Interamericano de Desarrollo (BID), la Superintendencia de Servicios Domiciliarios y el Sistema Único de información de servicios públicos domiciliarios (SUI). Asimismo, se incluyen cotizaciones de los sistemas fotovoltaicos provenientes de empresas del sector, así como cotizaciones de varios componentes en mercadolibre.com. De esta forma, se puede calcular el costo total de un sistema teniendo en cuenta el costo de oportunidad de reducir el uso de fuentes tradicionales. Luego, se comparan estos costos con los identificados previamente por Morales para el año 2011 (2013a), y para el año 2013 (2013b) de esta forma identificar la tendencia que se ha presentado en el mercado colombiano. Posteriormente, usando los datos arrojados por el software Homer, se calcula la generación del sistema representativo detallando los excedentes de energía comercializables y el consumo que se demanda desde el sistema interconectado. Finalmente, se procede a calcular el valor presente de neto de la inversión en un sistema fotovoltaico y el valor de la tarifa de alimentación necesaria para que ese valor presente sea cero (Rigter y Vidican, 2010). Los supuestos generales para el cálculo de la tarifa de alimentación se encuentran en la tabla 1. Es importante resaltar que la tarifa de alimentación calculada considera únicamente los costos y beneficios del posible adoptante, es decir, se trata de una valoración privada, y no incluye costos ni beneficios sociales. 
Tabla 1. Supuestos generales

\begin{tabular}{l|l|l}
\hline \multicolumn{1}{c}{ Parámetro } & \multicolumn{1}{c}{ Valor } & \multicolumn{1}{c}{ Fuente } \\
\hline Tasa de interés $(i)$ & $18 \%$ & Banco de la República (2017) \\
Maduración del proyecto $(M)$ & 25 años & Morales (2013a) \\
Porcentaje de cuota inicial () & $20 \%$ & Morales (2013a) \\
Excedente vendido a la red & $193,3 \mathrm{kWh}$ & Cálculos en Homer \\
Compras a la red & $304,7 \mathrm{kWh}$ & Cálculos en Homer \\
Año base IPC & 2008 & Banco de la República (2017) \\
Consumo promedio residencial & $200 \mathrm{kWh}$ & Gómez (2015)
\end{tabular}

Fuente: elaboración propia.

Se considera una instalación de $3 \mathrm{~kW}$ de potencia ya que este sistema está en capacidad de proveer más del 90\% de la demanda de un hogar típico en un país como Colombia la cual es de 200 kWh por mes. Además, este tamaño permite realizar comparaciones con estudios similares realizados previamente por el autor. La instalación típica que aquí se analiza se compone entonces de paneles solares, un inversor encargado de convertir la energía de corriente continua (DC) generada por los paneles, en corriente alterna (AC) la cual es la que se usa en la industria, el comercio y los hogares. Dado que el inversor tiene una vida útil estimada de 15 años, se debe considerar el valor de la reposición de este componente.

La decisión de adoptar o no la instalación fotovoltaica pasa por considerar el valor presente neto de adoptar la tecnología $\left(V P N_{A}\right)$ el cual debe ser mayo a cero (Rigter y Vidican, 2010). Este valor se compone de cuatro factores: $C I$; (i) la cuota inicial SC, (ii) el valor presente de la sucesión de cuotas mensuales para pagar el saldo restante SC, (iii) el valor presente de los costos evitados por el menor uso de la tecnología convencional $C E$ y (iv) el valor presente los ingresos por la venta de excedentes de energía VE. Suponiendo una tasa de interés $i$ y un horizonte de tiempo de M periodos, la fórmula queda 


$$
V P N_{A}=C I+\sum_{t=1}^{M} S C(1+i)^{M}+\sum_{t=1}^{M} C E(1+i)^{M}+\sum_{t=1}^{M} V E(1+i)^{M}
$$

El costo total de la instalación se puede definir como $C$, el cual se puede descomponer en dos fracciones. La primera es la cuota inicial CI la cual es un porcentaje 6 del costo total y la segunda es el saldo $(1-\theta)$ C que se amortizará mediante una sucesión de $M$ cuotas. Esta sucesión se representa mediante la fórmula

$$
S C=(1+(M-t+1) i)(1-\theta) \mathrm{CM}^{-1}
$$

El valor presente de los costos generados por usar la energía tradicional corresponde al valor de la energía de fuente convencional que se deja de consumir y está definido como el valor presente de la energía convencional $E$ multiplicada por la tarifa de esta fuente $T$. Los ingresos por la venta de excedentes VE se calculan multiplicando el valor de pagado por kilovatio generado FIT por la cantidad de energía liberada a la red $E$.

Es importante resaltar cómo esta medición del valor presente supone que los individuos solo consideran explícitamente sus costos y beneficios privados, pero no tiene en cuenta las externalidades que se generan frente a esta decisión. Sin embargo, es posible incorporar incentivos diseñados para tener en cuenta los costos y beneficios sociales. Algunos incentivos que se pueden considerar son un descuento en el valor total de la instalación, un precio preferencial pagado por los excedentes de energía o una tasa de interés menor en el financiamiento.

\section{Cálculo de costos y de tarifas de alimentación}

Considerando el costo de una instalación de $3 \mathrm{~kW}$ de potencia se encuentra que su costo es de 7.202 dólares (tabla 2). Para poder comparar con los resultados de dos estudios previos de Morales (2013a, 2013b), se comparan los costos de instalación con pesos constantes de 2008. También se calcula la evolución del número de salarios mínimos necesarios para pagar una instalación fotovoltaica. Para realizar este cálculo, se toma como referencia el Salario mínimo 
mensual legal vigente (SMMLV) en Colombia para los años 2011, 2013 y 2017.

Tabla 2. Costo de los componentes de un sistema de $3 \mathrm{~kW}$ de potencia (dólares)

\begin{tabular}{|c|c|c|c|c|}
\hline Componente & $\begin{array}{l}\text { Precio } \\
\text { unitario }\end{array}$ & $\begin{array}{c}\text { Canti- } \\
\text { dad }\end{array}$ & $\begin{array}{c}\text { Total } \\
\text { componente }\end{array}$ & $\begin{array}{c}\% \text { del } \\
\text { costo total }\end{array}$ \\
\hline Panel solar policristalino 250W & $\$ 213,3$ & 15 & $\$ 3.200,0$ & 44,4 \\
\hline Baterías 12V/205Ah & $\$ 366,7$ & 3 & $\$ 1.100,0$ & 15,3 \\
\hline Inversor 5200W, 120V & $1.527,3$ & 1 & $\$ 1.527,3$ & 21,2 \\
\hline Reposición Inversor & $\$ 127,6$ & 1 & $\$ 127,6$ & 1,8 \\
\hline Regulador PWM 12/24V 60A & $\$ 246,7$ & 3 & $\$ 740,0$ & 10,3 \\
\hline Materiales BOS & $\$ 99,7$ & 1 & $\$ 99,7$ & 1,4 \\
\hline $\begin{array}{l}\text { Instalación, operación y } \\
\text { mantenimiento }\end{array}$ & $\$ 407,7$ & 1 & $\$ 407,7$ & 5,7 \\
\hline $\begin{array}{l}\text { COSTO TOTAL DEL } \\
\text { SISTEMA }\end{array}$ & & & $\$ 7.202,3$ & 100,0 \\
\hline
\end{tabular}

Fuente: elaboración propia con datos de Vivasolar.com (2017), Gómez et al. (2016), Morales (2013a) y Morales (2013b).

Al analizar el periodo 2011-2017 (gráfico 1) se evidencia que el valor de las instalaciones ha venido bajando ya sea que se mida usando precios constantes o salarios mínimos necesarios. Esta tendencia es coherente con la que se ha visto en resto del mundo y que se explica por la pronunciada curva de aprendizaje que ha caracterizado al mercado fotovoltaico en un periodo de más de 30 años y que ha permitido que cada vez que se duplique la producción mundial se reduzca el precio en un 18\% (Haysom, Jafarieh, Anis, Hinzer \& Wright, 2015). Asimismo, se evidencia que ha sido mayor la reducción en términos de los salarios mínimos requeridos para comprar una instalación fotovoltaica, lo cual indica que la reducción en el precio viene acompañada de una mejora en la capacidad adquisitiva para comprar este tipo de tecnologías.

También se pueden analizar estos resultados comparando el periodo 2011-2013 y el periodo 2013-2017. Durante el primer periodo se encuentra una reducción del 43,6\% en el costo de las instalaciones y del $46,5 \%$ en el número de salarios requeridos. Sin embargo, en el periodo 2013-2017 la tasa de reducción ha sido menor: en el caso del costo, este se redujo en $21,2 \%$ mientras que el número de sa- 
larios requeridos se redujo en un 19,5\%. La razón para esta menor reducción en el precio radica principalmente en la devaluación de la tasa de cambio, la cual ha implicado un mayor costo de los productos importados desde Colombia. Una tendencia similar se evidencia en el mercado global de instalaciones fotovoltaicas señalada por el Consejo Mundial de Energía (WEC, por sus siglas en inglés): se ha presentado una menor tasa de reducción en el precio en el periodo 2013 - 2014 debida presumiblemente a la caída en los precios del petróleo, lo cual ha implicado menores inversiones en generación usando energías renovables (WEC, 2016).

Gráfico 1. Costo de un sistema fotovoltaico de $3 k W$ en Colombia durante el periodo 2011-2017 en millones de pesos y Salarios mínimos legales vigentes (SMMLV)

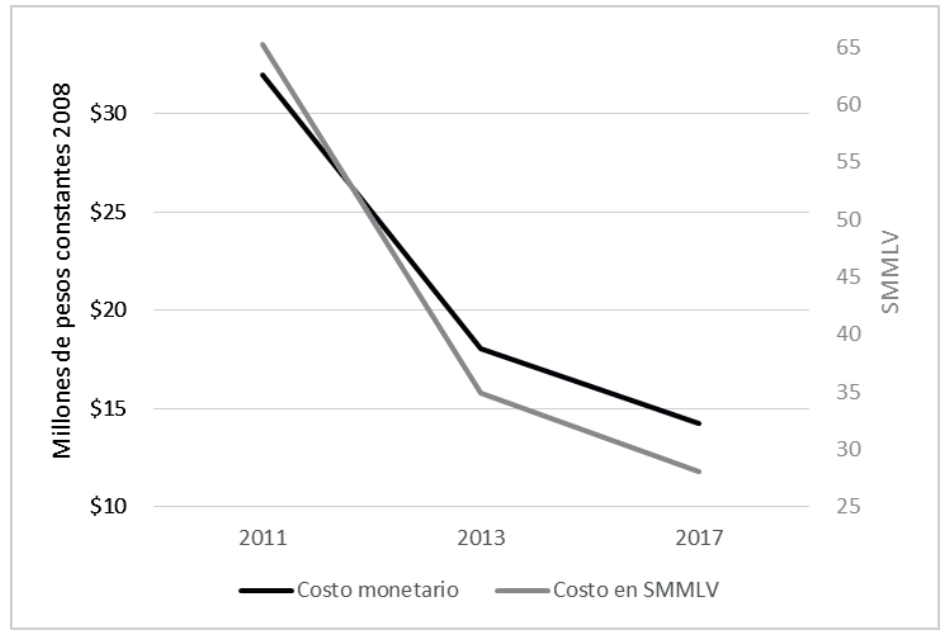

Fuente: Elaboración propia con datos de Morales (2013a), Morales (2013b) y Vivasolar (2017)

Respecto a la tarifa de alimentación, se encuentra que para el caso de Bogotá, se requiere una tarifa de alimentación de 0,209 dólares el kilovatio hora (626 pesos suponiendo una tasa de cambio de 3000 pesos por dólar) para que el valor presente neto de la adopción sea cero. Este resultado no es comparable con los obtenidos por Morales (2013a, 2013b) ya que en este caso se tiene en cuenta el costo evitado por la menor compra de energía al sistema interconectado. 
En el caso de San Andrés, la tarifa de alimentación calculada es de 0,213 dólares kilovatio hora (638 pesos), la cual resulta mayor que la de Bogotá porque por cuenta del subsidio del Estado, los habitantes en San Andrés pagan una menor tarifa por kilovatio hora. Esto implica que el costo de oportunidad por reducir el uso de la fuente convencional es mayor en San Andrés que en Bogotá, lo cual se traduce en un menor incentivo a cambiar de fuente. Sin embargo, es importante señalar que el valor de esta tarifa de alimentación resulta menor que el costo unitario de la energía ofrecida en el departamento que según datos del SUI es de 0,277 dólares. Es decir, hay indicios para considerar que al Estado colombiano le resultaría más barato o por lo menos equivalente, subsidiar la generación de energía en San Andrés Islas bajo un esquema de tarifas de alimentación usando paneles fotovoltaicos, que continuar con el sistema tradicional de generación mediante diésel.

\section{Aportes conclusivos}

Aunque el costo de instalación en Bogotá ha bajado y por lo tanto, también ha bajado el valor de una eventual tarifa de alimentación, se encuentra que instalaciones residenciales de este tamaño, todavía no son costo-eficientes. El costo de la energía tradicional, es decir, la ofrecida en el sistema interconectado sigue siendo considerablemente menor a las de los sistemas fotovoltaicos estudiados. No obstante, es posible considerar que sistemas de mayor tamaño, instalados en, los sectores comercial o industrial, o en propiedad horizontal, pueden ofrecer mejores resultados.

Por el contrario, el caso del departamento de San Andrés y Providencia muestra resultados prometedores, aunque no exentos de retos. Dado que todos los usuarios de energía en el departamento tienen subsidio, hay pocos incentivos privados a la adopción de energías renovables. No obstante, los resultados de este trabajo muestran que el Estado y en últimas el conjunto de la sociedad, si podría tener beneficios directos asociados a la reducción en los costos de generación. Esto sin entrar a considerar otros posibles beneficios ambientales y sociales, derivados de las menores emisiones de gases de efecto invernadero, la mejora en la confiabilidad en la prestación del servicio o los empleos que se pueden obtener en un mercado de instalación y mantenimiento de sistemas fotovoltaicos. Por ende, el diseño de la política energética en el país, pero especialmente en San 
Andrés, debe buscar una mayor armonía entre el interés individual y el colectivo.

Es necesario que el proceso de adopción de estos sistemas se haga con la participación de las comunidades del departamento para que ellas mismas contribuyan en el diseño de la estructura de generación. De esta forma, las mismas comunidades participarían en la identificación y mitigación de los problemas que puedan aparecer, así como en el aprovechamiento de los beneficios. Este involucramiento posibilita que el sistema energético del departamento evolucione de forma acorde a las necesidades de la región. De hecho, los esquemas de generación distribuida ofrecen la capacidad de contribuir en ese mayor involucramiento de la sociedad civil, ya que le ofrecen al ciudadano la posibilidad de diseñar un esquema que rentabilice su inversión (Rifkin, 2014).

En la medida en que el esquema de generación distribuida depende menos de la iniciativa de entidades de gobiernos con serios problemas de gestión y le dan más autonomía al usuario, permiten que el sistema energético de una región se gestione de forma descentralizada, aumentando su capacidad para procesar información y adaptarse a las circunstancias del entorno. Las tecnologías de generación distribuida se benefician de tecnologías de la información y las comunicaciones (TIC) y por lo tanto, posibilitan una mejor coordinación entre los integrantes del sistema energético. Resulta necesario, diseñar herramientas acordes al contexto del país que faciliten la adopción de estos sistemas. Estas herramientas de simulación, cálculo y coordinación, deben ser de uso fácil y con carácter social de manera que faciliten la demostración, la toma de decisiones y el posterior seguimiento a las instalaciones. En definitiva, la adopción de esquemas de energía fotovoltaica con generación distribuida representa una plataforma para estimular la innovación con impacto local.

\section{Bibliografía}

Acemoglu, D., Johnson, S., \& Robinson, J. A. (2005). Institutions as a fundamental cause of long-run growth. Handbook of economic growth, 1, 385-472. 
Bass, F. (1969). A new product growth for model consumer durables. 15(5): Management Science, 15(5), 215-227.

Berkun, S. (2010). The myths of innovation." O'Reilly Media, Inc.".

Compañía Expertos en Mercados (XM). (2017). Capacidad efectiva neta. [online] Available at: http://informesanuales.xm.com.co/2015/SitePages/operacion/2-6-Capacidad-efectiva-neta.aspx

Contraloría General de la República. (2012). Informe de auditoría al proyecto contrato de concesión con exclusividad para la prestación del servicio de energía eléctrica en el área geográfica de San Andrés, Providencia y Santa Catalina $n^{\circ}$ 067. Recuperado de http://bit. ly/2vN3tec

Dahlman, C. J. (1979). The problem of externality. The journal of law and economics, 22(1), 141-162.

Dinero. (2017). San Andrés le da ejemplo a Electricaribe: ¡Sí se puede! [online] Disponible en: http://bit.ly/2eMiTYH

Gómez, J. R., Afanador, J. E., \& Rodríguez, J. L. (2016). Hacia la sostenibilidad eléctrica en el Archipiélago de San Andrés, Providencia y Santa Catalina, Colombia: Análisis de alternativas. Recuperado del sitio de internet del Banco Interamericano de Desarrollo: http://bit. ly/2glGSFc

Graziano, M., \& Gillingham, K. (2014). Spatial patterns of solar photovoltaic system adoption: the influence of neighbors and the built environment. Journal of Economic Geography, 15(4), 815-839.

Guevara-Stone, L., Hawley, K., \& Tubb, A. (2016). San Andrés phase 1 visioning workshop. Recuperado del sitio de internet de Rocky Mountain Institution: http://bit.ly/2f5jlhC

Haysom, J. E., Jafarieh, O., Anis, H., Hinzer, K., \& Wright, D. (2015). Learning curve analysis of concentrated photovoltaic systems. Progress in Photovoltaics: Research and Applications, 23(11), 1678-1686.

IDEAM, PNUD, MADS, DNP, CANCILLERÍA. (2016). Inventario nacional y departamental de Gases Efecto Invernadero - Colombia. Ter- 
cera Comunicación Nacional de Cambio Climático. Bogotá D.C., Colombia.

Intergovernmental Panel on Climate Change (IPCC). (2014). Climate Change 2014-Impacts, Adaptation and Vulnerability: Regional Aspects. Cambridge University Press.

International Energy Agency (IEA). (2014). World Energy Outlook.

Kahneman, D. (2011). Thinking, fast and slow. Macmillan.

Kerr, W. R., Nanda, R., y Rhodes-Kropf, M. (2014). Entrepreneurship as experimentation. The Journal of Economic Perspectives, 28(3), $25-48$.

McCartney, M. (2009). Living with dams: managing the environmental impacts. Water Policy, 11(S1), 121-139.

Morales Sánchez, C. F. (2013a). Cálculo de una tarifa de alimentación para instalaciones fotovoltaicas residenciales en Colombia. Semestre Económico, 16(34).

Morales Sánchez, C. F. (2013b). Posibilidades y retos de un mercado de micro-generación de energía limpia a través de paneles fotovoltaicos en Colombia. Universidad Nacional. Bogotá, Colombia

Morrison, K., \& Potts, J. (2008). Toward behavioural innovation economics-Heuristics and biases in choice under novelty (No. 379).

Ochoa Urrego, R. L., \& Peña Reyes, J. I. (octubre, 2012) Teoría de la Difusión de Innovaciones: Evolución y uso en los Sistemas de Información. Ponencia presenta en el III Congreso Internacional de Gestión Tecnológica e Innovación COGESTEC, Medellín, Colombia.

Paredes, J., Ramírez J. (2017). Energías renovables variables y su contribución a la seguridad energética: complementariedad en Colombia. Recuperado del sitio de internet del Banco Interamericano de Desarrollo: http://bit.ly/2xQaJDd 
Perez, C. (2004). Technological revolutions, paradigm shifts and socio-institutional change. Globalization, economic development and inequality: An alternative perspective, 217-242.

Photovoltaic-software.com. (2017). Online photovoltaic software for professional: PV solar energy calculator and cost benefits simulator, sizing of solar photovoltaic systems, SOLARGIS, Polysun online, Solardesign, EASYSOLAR. [en línea] Disponible en: http:// photovoltaic-software.com/online-professional.php

Rifkin, J. (2014). The zero marginal cost society: The internet of things, the collaborative commons, and the eclipse of capitalism. St. Martin's Press.

Rigter, J., \& Vidican, G. (2010). Cost and optimal feed-in tariff for small scale photovoltaic systems in China. Energy Policy, 38(11), 6989-7000.

Rogers, E. (2003). Diffusion of innovations. New York: Free Press.

Rusko, R. (2012). The redefined role of consumer as a prosumer: Value co-creation, coopetition. Production and Manufacturing System Management: Coordination Approaches and Multi-Site Planning, 162-174.

Saenz, J. (2017). El país despierta a las energías renovables. El Espectador. Recuperado de http://bit.ly/2gLDyge

Sandén, B. (2005). The economic and institutional rationale of PV subsidies. Solar energy, 78, 137-146.

Schumpeter, J. (1939). Business cycles. a theoretical, historical and statistical analysis of the capitalist process. Nueva York: McGraw-Hill Book Company.

Sistema Único de Información de Servicios Públicos Domiciliarios (SUI). (2017). Consolidado de información comercial ZNI. Obtenido de http://bit.ly/2vMZIFo

Sun, P., \& Nie, P. Y. (2015). A comparative study of feed-in tariff and renewable portfolio standard policy in renewable energy industry. Renewable Energy, 74, 255-262. 
Unidad de Planeación Minero Energética UPME. (2015). Integración de las energías renovables no convencionales en Colombia. Recuperado de: http://bit.ly/2w7WoQo

Vivasolar Colombia. (2017). Paneles solares baratos Colombia. [ en línea] Disponible en: https://www.vivasolar-colombia.com/ [Accessed 30 Jun. 2017].

Wold Energy Council (WEC). (2016). World Energy Resources 2016. Recuperado de http://bit.ly/2eL37cO 